\title{
The impact of major league baseball on the incidence of operative hand and facial trauma at a level 1 trauma center
}

\author{
Joseph A. Ricci ${ }^{1}$, Christina R. Vargas ${ }^{2}$, Olivia A. Ho ${ }^{1}$, Samuel J. Lin ${ }^{1}$, Bernard T. Lee ${ }^{1}$ \\ ${ }^{1}$ Division of Plastic and Reconstructive Surgery, Beth Israel Deaconess Medical Center, Harvard Medical School, Boston, MA; ${ }^{2}$ Department of \\ Plastic Surgery, University Hospitals Cleveland Medical Center, Case Western Reserve University, Cleveland, OH, USA
}

Background Professional affiliation between medical centers and professional sports teams can be mutually beneficial in the provision of healthcare and marketing strategy. Anecdotal evidence suggests a link between trauma volume and sporting events; however, there is limited data. This study aims to characterize the incidence of operative hand and facial trauma during professional baseball home games.

Methods A retrospective review of surgical cases for traumatic hand or facial injuries at a level 1 center between 1999 and 2012 was performed. Demographic information including date of injury, admission status, and operative repair were collected. Patients were grouped based on whether their trauma occurred on the date of a home game.

Results Operative hand and facial trauma occurred at a rate of 33.4 injuries per 100 days with home games, compared to 22.2 injuries per 100 days (incidence rate ratios, 1.50; 95\% confidence interval, 1.34-1.69). When home games were played, patients were more likely to present as a result of motorcycle accidents $(3.1 \%$ vs. $1.5 \%$; $P=0.04)$ or bicycle accidents $(5.0 \%$ vs. $2.6 \% ; P=0.01)$. Other mechanisms of trauma were not statistically different. There was an increase incidence of injuries during home games in August; weekly variability showed an increased incidence during the weekends.

Conclusions There was an increased rate of operative hand and facial injuries on dates with professional home games. The incidence of injuries during home games was higher in the late summer and on the weekends. Further analysis may allow improved resource allocation and strategies for injury prevention and treatment.

Keywords Baseball / Hand injuries / Facial injuries
Correspondence: Bernard T. Lee Division of Plastic and Reconstructive Surgery, Beth Israel Deaconess Medical Center, Harvard Medical School, 110 Francis St \#5A, Boston, MA 02215, USA

Tel: +1-617-632-7835

Fax: +1-617-632-7840

E-mail: blee3@bidmc.harvard.edu
This article was presented at the ASPS Meeting on September 23-27, 2016, in Los Angeles, CA, USA.

\section{INTRODUCTION}

Among healthcare professionals at trauma centers, there is a wide variety of generalizations about the timing of hand and fa- cial trauma volume over the course of a year. Most providers who have spent time in the hospital setting have heard phrases like "a full-moon Friday night" to describe superstitious beliefs that there will be a higher volume of patients during that shift 
directly related to factors like day of the week, among other things. Anecdotally in the United States, the weekends and the summertime are thought to be the busiest time for most types of trauma. Previous studies have evaluated the relationship between the number of trauma admissions and various factors like: temperature, precipitation, snow days, length of daylight, lunar phase, holidays, day of the week, and month of the year [15]. There is even some data to support that there is an increase in the number of deaths toward the beginning of each month, including vehicular crashes and violent penetrating trauma [6]. Still other studies have described an increased overall incidence of fractures, hand fractures, and burn admissions in the springtime, and hip and distal forearm fractures in the winter [7-13].

The inconsistency in daily, weekly, and monthly trauma volume can pose a significant challenge to administrators and hospitals arranging appropriate staff coverage for the emergency department, operating rooms, inpatient wards, and intensive care units. Access to descriptive data relevant to a medical center's characteristics could improve the allocation of resources and help to ensure adequate availability during peak period, but there is little published data investigating the presence or absence of important trends in operative trauma.

At the Beth Israel Deaconess Medical Center, anecdotal evidence among surgeons and employees suggests that there may be another factor, not previously described, which may additionally impact the volume of trauma: major league baseball home games. Our medical center is not only the official hospital for a professional baseball team, but our center is located a half mile from the stadium. Given the proximity of the stadium to our trauma center, the vast majority of injuries occurring within or near the stadium during home games are evaluated at our hospital. As a result, even in a major metropolitan area, it is possible that the impact of a professional sports team on the incidence of operative trauma could be significant.

To date, there has been little published data available describing the variable incidence of operative traumatic hand and facial injuries requiring plastic and reconstructive surgical intervention in the United States. Furthermore, correlation between cyclic variables such as weeks and months could serve as important predictors of plastic surgical operative volume, allowing for improvement in staffing consideration, vacation planning, resident scheduling, shift changes, and operating room resource allocation to improve quality and efficiency. In this study, we present the longest review of cases in a level 1 trauma center requiring reconstructive surgical intervention to date, aiming to characterize the variability of operative hand and facial trauma over a 14-year period, focusing on the effect of a professional baseball team.

\section{METHODS}

A retrospective review was conducted of all operative cases involving hand and facial trauma from January 1, 1999 through December 31, 2012 performed by the plastic and reconstructive surgery service at a tertiary medical center. Institutional Review Board (IRB) approval was secured for this study (IRB Protocol No. 2011P00041). Case start date, procedure, and de-identified patient demographic data were populated from electronic operating room records. Additional data collected were date of injury, admission date, discharge date (if applicable), trauma mechanism, gender, age, insurance status, and state of residence. Surgery performed more than 30 days after the date of trauma was excluded. Multiple procedures for the same patient following traumatic injury were included if they occurred within 30 days of the injury. Cases included inpatient and outpatient surgeries and ranged from emergent to elective.

Inpatient status indicated those patients who were admitted at the time of surgery. Insurance status was collected as a binary variable: any public or private coverage at the time of presentation was included. State of residence refers to the patient's home billing address at the time of surgery. Traumatic mechanism was subdivided into one of several categories: motor vehicle accident, motorcycle crash, bicyclist crash, pedestrian struck, fall, assault, industrial/mechanical, animal bite/attack, gunshot wound, sports, and unknown/other. Many patients in the "other" category had sustained injuries from doors, kitchen knives, furniture, and similar incidents at home.

In order to assess the relationship with home professional baseball games, the historical season schedules were obtained for our local professional baseball team, for the 1999 to 2012 seasons. Data collected included the outcome of the game (win or loss), the score differential, the opponent (division rival or not) and whether it was a playoff game. Patients were categorized into groups based on whether their trauma occurred on the date of a home game. Descriptive analysis of the variability in injury volume was performed for cases overall as well as for hand and facial trauma subgroups. Statistical analysis was performed using chi-square tests of observed versus expected event frequencies. A P-value of less than 0.05 was considered statistically significant.

\section{RESULTS}

Our facility is an accredited Level 1 Trauma Center in Boston, Massachusetts, with 649 inpatient beds and 77 critical care beds [14]. From the most recent data, there were a total of 55,677 emergency department visits and 548,677 outpatient visits in 
2012 [15]. Over the span of the study period, there were approximately 20,000 operative cases performed overall by the plastic surgeons at our institution.

A total of 382 cases occurred as a result of injuries sustained on the dates of home games for our local baseball team. The victims were $77 \%$ male $(n=297)$ and $23 \%$ female $(n=85)$. There were a total of 249 operative hand traumas $(65 \%)$ and 133 operative facial traumas (35\%) over that span (Table 1). Of all the cases, 114 (29.8\%) were performed during an inpatient admission and these patients had an average length of stay of 5 days (range, 1-40 days). The average time from injury to surgery was 8.2 days (range, $0-81$ days). As shown in Table 1, the most frequently observed traumatic mechanisms for all cases were industrial or mechanical injuries (17.5\%), falls (18\%), and assault (17\%). Patients who were injured on the dates of home games tended to have a similar insurance status ( $88 \%$ covered) compared to patients who were injured on dates when there was not

Table 1. Plastic and reconstructive hand and facial trauma during major league baseball home games

\begin{tabular}{|lccc|}
\hline Variable & $\begin{array}{c}\text { Injuries on } \\
\text { baseball home } \\
\text { game dates }\end{array}$ & $\begin{array}{c}\text { Injuries on } \\
\text { other dates }\end{array}$ & P-value \\
\hline Total no. & 382 & 1,155 & \\
Sex & & & NS \\
Male & $297(78)$ & $862(75)$ & \\
Female & $85(22)$ & $293(25)$ & \\
Average age (yr) & 37.9 & 39.6 & NS \\
Trauma type & & & \\
$\quad$ Hand & $249(65)$ & $732(63)$ & NS \\
$\quad$ Facial & $133(35)$ & $423(37)$ & NS \\
Admission status & & & \\
$\quad$ Inpatient & $114(30)$ & $377(33)$ & NS \\
Outpatient & $268(70)$ & $778(67)$ & NS \\
Average length of stay (day) & 5 & 6 & NS \\
Mechanism of injury & & & \\
$\quad$ Motor vehicle accident & $35(9)$ & $125(11)$ & NS \\
Motorcycle crash & $12(3)$ & $17(2)$ & 0.049 \\
Cyclist & $19(5)$ & $26(2)$ & 0.012 \\
Pedestrian struck & $10(3)$ & $32(3)$ & NS \\
Fall & $63(16)$ & $213(18)$ & NS \\
Assault & $67(18)$ & $198(17)$ & NS \\
Industrial \& mechanical injury & $67(18)$ & $234(20)$ & NS \\
Animal & $2(0.5)$ & $10(0.9)$ & NS \\
Penetrating trauma & $2(0.5)$ & $7(0.6)$ & NS \\
Sports & $39(10)$ & $94(8)$ & NS \\
Other & $66(17)$ & $199(17)$ & NS \\
Insurance status & & & \\
Insured & $338(88)$ & $1,038(90)$ & NS \\
$\quad$ Not insured & $44(12)$ & $117(10)$ & NS \\
Residence & & & \\
Massachusetts & $346(91)$ & $1,006(87)$ & NS \\
Other state & $36(9)$ & $149(13)$ & NS \\
\hline Values are presented as number (\%). & & & \\
NS, not significant. & & & \\
\hline
\end{tabular}

a home game ( $88 \%$ vs. $87 \%$ covered; $\mathrm{P}=0.26$ ) as well as the entire cohort of patients (90\%).

Operative trauma requiring reconstructive interventions occurred at a rate of 33.4 injuries per 100 days when home games were played, compared to 22.2 injuries per 100 days when the team did not have a home game (incidence rate ratio, 1.50; $95 \%$ confidence interval, 1.34-1.69). Similar trends were noted when subgroup analysis was performed individually for hand or facial trauma (Table 2). Additionally, when the groups were compared, patients were more likely to present as a result of motorcycle accidents ( $3.1 \%$ vs. $1.5 \% ; \mathrm{P}=0.04$ ) or bicycle accidents ( $5.0 \%$ vs. $2.6 \%$; $\mathrm{P}=0.01$ ) when home games were played. Other mechanisms of trauma, age, gender, insurance status, need for admission, and length of stay were not statistically different between the groups. When comparing home games that resulted in wins or losses, there were no differences in the incidence of injuries (32.1 injuries per 100 days vs. 32.3 injuries per 100 days). In addition, when examining home games against division rivals, there was a slight decrease in incidence of injuries to 19.0 per 100 days. Finally, home games during the playoffs resulted in 25.9 injuries per 100 days.

Monthly variability was evaluated during the baseball season over a 6-month period from April to September (7 months if playoff games extended into October) (Fig. 1). There was an even distribution of injuries within those months with an average of 54.5 injuries per month. The highest number of injuries was recorded in August $(\mathrm{n}=81)$ and October had the lowest $(\mathrm{n}=$ 11). On a weekday basis, there were an average of 62 injuries per day of the week. Friday $(n=74)$, Saturday $(n=66)$ and Sunday $(n=63)$ had the highest numbers of injuries compared to the other days of the week (Fig. 2).

\section{DISCUSSION}

Among healthcare professionals at trauma centers, there is a wide variety of generalizations about the timing of hand and facial trauma volume over the course of a year. To date, there have

Table 2. A comparison of the rates of operative trauma on home game dates versus non-home game dates

\begin{tabular}{|lccc|}
\hline & $\begin{array}{c}\text { All } \\
\text { dates }\end{array}$ & $\begin{array}{c}\text { Baseball } \\
\text { home game } \\
\text { dates }\end{array}$ & $\begin{array}{c}\text { Non-baseball } \\
\text { home game } \\
\text { dates }\end{array}$ \\
\hline No. of Injuries & 1,537 & 382 & 1,155 \\
Rate of operative trauma overalla) $^{\text {Rate of operative hand trauma }}$ & 29.5 & 33.4 & 22.2 \\
Rate of operative face trauma & 18.8 & 21.9 & 14.0 \\
\hline Rates listed as injuries per 100 days. & 10.6 & 11.7 & 8.1 \\
\hline Rated & & \\
\hline
\end{tabular}




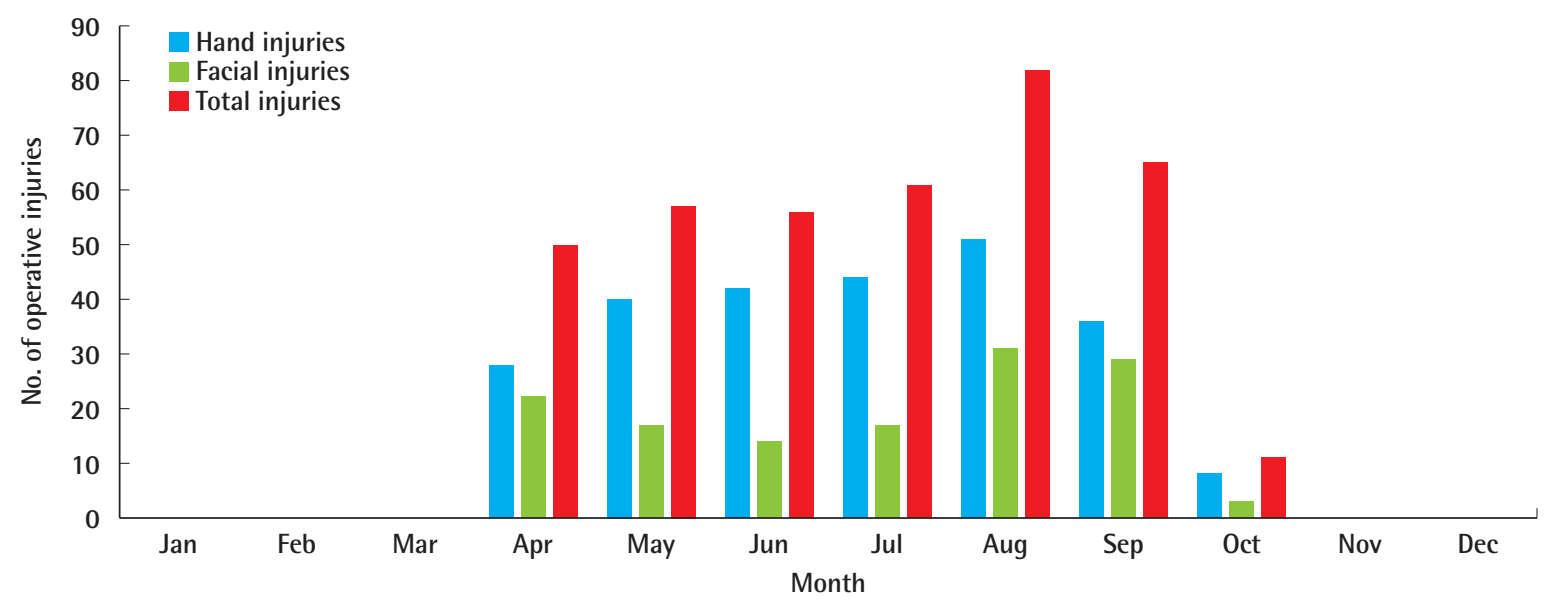

Fig. 2. Weekday variation in trauma during baseball games

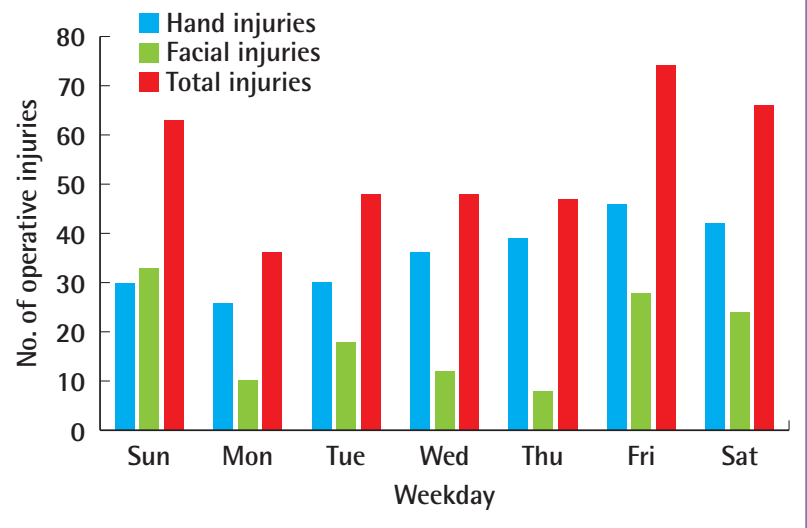

been no other reports in the literature evaluating the impact of a home game for a professional sports team on the incidence of trauma. As our medical center is the official hospital of a professional baseball team and is also located in close proximity to the stadium $(0.8 \mathrm{~km})$, a majority of the traumatic injuries occurring before, during, and after the game are evaluated and treated at our facility. There have been anecdotal reports from staff at our facility of increased trauma volume during baseball home games due to the increased pedestrian traffic, increased vehicular traffic, alcohol consumption, and huge crowds that are associated with home games. In this study, the rate of operative trauma requiring reconstructive interventions increased by $50 \%$ when major league home games were played compared to when the team did not have a home game. Similar trends were noted when subgroup analysis was performed individually for hand or facial trauma.
Despite the difference in the rates of operative plastic surgical trauma, there was surprisingly little difference when the mechanisms of injury were compared between patients who were injured on days when there were baseball home games and those injured on dates when there was not a baseball game. Industrial and mechanical injury, assault, and falls represented the most commonly seen operative trauma, however, these mechanisms did not increase during home games. There was also no difference in the rate of sports related trauma-perhaps suggesting that the increased rate of injury was not related to players being injured but rather to fans being injured, as hypothesized. The only difference in the mechanism of injury between the groups was with respect to motorcycle and bicycle accidents. It is possible that increased vehicular traffic at game time make it more dangerous for those on bikes and motorcycles.

Anecdotally, among providers, sporting events during the weekends and summer months are thought to be the busiest time for most types of trauma. In this study, we found this to be true. During baseball home games the highest operative hand and facial trauma volume occurred in the late summer (August) and on weekends (Friday to Sunday). This was consistent for both hand and facial injuries.

Previously published reviews of traumatic injuries incidence have presented a wide array of conflicting results, with little consensus on the data. For instance, a study evaluating operative maxillofacial fractures in the Netherlands found a peak incidence in the springtime and on weekends [16]. A similar review of craniomaxillofacial trauma from Austria noted the highest incidence in January, followed by August, then February, with November having the lowest incidence; while reports from Brazil found the highest frequency of injuries to occur in the sum- 
mer $[17,18]$. The incidence of pediatric facial fractures has also been studied in several countries, again with little consensus in the results [19-21]. It seems that perhaps on a global scale, the forces driving variability in trauma volume are impacted by local, regional, and national factors more so than the day of the week or month of the year.

In 2001, a study from another local trauma center found a significant relationship between weather and general trauma admissions [2]. In addition to the meteorological association, the study also demonstrated a peak in the overall trauma incidence in the months of July and August, as well as on Saturday and Sunday, when compared to other days of the week during their 6-year review. Importantly, the incidence pattern closely mirrored our findings, which demonstrated the hand trauma subgroup to have a similar distribution with respect to months and days of the week. This suggests that while some injury types (hand injuries) may follow the general trend of overall trauma volume, others, like facial trauma, do not. This observation may also help to explain the variety of trends published in the trauma and subspecialty literature noted previously.

There are several unique characteristics of trauma cases requiring plastic and reconstructive surgery involvement. Plastic and reconstructive surgeons treat a range of injuries requiring emergent surgical intervention (i.e., amputated body part requiring replantation or revascularization); urgent intervention (i.e., open fractures or severe burns); semi-elective intervention (i.e., some facial fractures or isolated tendon lacerations); and delayed intervention (cosmetic reconstruction). In addition, not all injuries which will require plastic surgical intervention are evaluated at the time of presentation to the emergency room during trauma activations. Many of these injuries are identified on secondary or tertiary survey after other, immediately lifethreatening injuries have been addressed by the trauma team. Variation in the time to consultation of specialist services, like plastic surgery, can contribute to the difficulty in predicting demand for these resources. In contrast to trauma staff, the plastic surgery staff at most centers are available when on call, but are not required to be in house, further compounding the importance of anticipating periods of high volume. Many patients who require plastic surgical intervention will also require prolonged follow up, hardware removal, staged reconstruction, or late revision, placing increased demand on outpatient specialty clinics.

There are several limitations to this study. First, as a retrospective review, there is the potential for inadvertent bias with regard to medical record review for data collection. Injuries requiring plastic and reconstructive procedures performed outside of the operating room (e.g., in the emergency department or outpa- tient clinic) were not included. Our institution does not routinely treat pediatric patients for traumatic injury, limiting the generalizability of these conclusions to adult trauma populations. Finally, this is a single institution study in a metropolitan academic level 1 trauma center with four distinct meteorological seasons, and as such, the patterns identified here may not apply to small centers in other cities or climates.

Overall, the results of this study demonstrated an increased rate of operative hand and facial injuries on dates when professional baseball home games were played. The incidence of injuries during home games was higher in the late summer and on the weekends. While not all trauma centers may be located in such close proximity to professional baseball stadiums, the results of this study should encourage hospital leadership to consider the timing of local community events to improve resource allocation and strategies for injury prevention and treatment. The correlation between cyclic variables such as weeks and months should serve as important predictors of plastic surgical operative volume, allowing for improvement in staffing consideration, vacation planning, resident scheduling, shift changes, and operating room resource allocation to improve quality and efficiency.

\section{NOTES}

\section{Conflict of interest}

No potential conflict of interest relevant to this article was reported.

\section{Ethical approval}

The study was approved by the Institutional Review Board of the Beth Israel Deaconess Medical Center (IRB No. 2011P 00041) and performed in accordance with the principles of the Declaration of Helsinki. Written informed consent was waived.

\section{Author contribution}

Ricci JA conceptualized this manuscript, performed data analysis and performed writing and editing of this manuscript. Vargas CR performed data collection, data analysis and editing of this manuscript. Ho OA performed data collection and editing of this manuscript. Lin SJ performed conceptualization of this protect, oversight of data acquisition and editing of this manuscript. Lee BT performed conceptualization of this manuscript, oversight of data collection, project administration and editing of this manuscript.

\section{ORCID}

Joseph A. Ricci https://orcid.org/0000-0002-8433-6446 
Olivia A. Ho https://orcid.org/0000-0002-7477-0855

Samuel J. Lin https://orcid.org/0000-0003-2698-2658

Bernard T. Lee https://orcid.org/0000-0002-5533-3166

\section{REFERENCES}

1. Weston-Simons J, Jack CM, Doctor C, et al. The impact of snow on orthopaedic trauma referrals. Injury 2012;43:10336.

2. Bhattacharyya T, Millham FH. Relationship between weather and seasonal factors and trauma admission volume at a level I trauma center.J Trauma 2001;51:118-22.

3. Atherton WG, Harper WM, Abrams KR. A year's trauma admissions and the effect of the weather. Injury 2005;36:406.

4. Rising WR, O'Daniel JA, Roberts CS. Correlating weather and trauma admissions at a level I trauma center. J Trauma 2006;60:1096-100.

5. Stomp W, Fidler V, ten Duis HJ, et al. Relation of the weather and the lunar cycle with the incidence of trauma in the Groningen region over a 36-year period. J Trauma 2009;67: 1103-8.

6. Phillips DP, Christenfeld N, Ryan NM. An increase in the number of deaths in the United States in the first week of the month: an association with substance abuse and other causes of death. N Engl J Med 1999;341:93-8.

7. van Onselen EB, Karim RB, Hage JJ, et al. Prevalence and distribution of hand fractures. J Hand Surg Br 2003;28:4915.

8. Feehan LM, Sheps SB. Incidence and demographics of hand fractures in British Columbia, Canada: a population-based study. J Hand Surg Am 2006;31:1068-74.

9. Hultman CS, Tong WT, Surrusco M, et al. To everything there is a season: impact of seasonal change on admissions, acuity of injury, length of stay, throughput, and charges at an accredited, regional burn center. Ann Plast Surg 2012;69: 30-4.

10. Jacobsen SJ, Sargent DJ, Atkinson EJ, et al. Population-based study of the contribution of weather to hip fracture seasonality. Am J Epidemiol 1995;141:79-83.

11. Jacobsen SJ, Sargent DJ, Atkinson EJ, et al. Contribution of weather to the seasonality of distal forearm fractures: a population-based study in Rochester, Minnesota. Osteoporos Int 1999;9:254-9.

12. Parsons N, Odumenya M, Edwards A, et al. Modelling the effects of the weather on admissions to UK trauma units: a cross-sectional study. Emerg Med J 2011;28:851-5.

13. Masterson E, Borton D, O'Brien T. Victims of our climate. Injury 1993;24:247-8.

14. American College of Surgeons. Verified trauma centers [Internet]. Chicago, IL [cited $2015 \mathrm{Nov}$ 14]. Available from http://www.facs.org/trauma/verified.html.

15. Beth Israel Deaconess Medical Center. Stats and facts [Internet]. Boston, MA [cited $2015 \mathrm{Nov}$ 14]. Available from https://www.bidmc.org/about-bidmc.

16. van Hout WM, Van Cann EM, Abbink JH, et al. An epidemiological study of maxillofacial fractures requiring surgical treatment at a tertiary trauma centre between 2005 and 2010. Br J Oral Maxillofac Surg 2013;51:416-20.

17. Kraft A, Abermann E, Stigler R, et al. Craniomaxillofacial trauma: synopsis of 14,654 cases with 35,129 injuries in 15 years. Craniomaxillofac Trauma Reconstr 2012;5:41-50.

18. Paes JV, de Sa Paes FL, Valiati R, et al. Retrospective study of prevalence of face fractures in southern Brazil. Indian J Dent Res 2012;23:80-6.

19. Gassner R, Tuli T, Hachl O, et al. Craniomaxillofacial trauma in children: a review of 3,385 cases with 6,060 injuries in 10 years. J Oral Maxillofac Surg 2004;62:399-407.

20. Eggensperger Wymann NM, Holzle A, Zachariou Z, et al. Pediatric craniofacial trauma.J Oral Maxillofac Surg 2008;66: 58-64.

21. Nardis Ada C, Costa SA, da Silva RA, et al. Patterns of paediatric facial fractures in a hospital of São Paulo, Brazil: a retrospective study of 3 years. J Craniomaxillofac Surg 2013;41: 226-9. 